\title{
Surfaces with maximal constant mean curvature
}

\author{
Jan Metzger \\ jan.metzger@aei.mpg.de \\ Albert-Einstein-Institut, Am Mühlenberg 1, D-14476 Potsdam, Germany.
}

\begin{abstract}
In this note we consider asymptotically flat manifolds with non-negative scalar curvature and an inner boundary which is an outermost minimal surface. We show that there exists an upper bound on the mean curvature of a constant mean curvature surface homologous to a subset of the interior boundary components. This bound allows us to find a maximizer for the constant mean curvature of a surface homologous to the inner boundary.

With this maximizer at hand, we can construct an increasing family of sets with boundaries of increasing constant mean curvature. We interpret this familiy as a weak version of a CMC foliation.
\end{abstract}

\section{Introduction}

Consider a non compact three dimensional Riemannian manifold $(M, g)$ with compact interior boundary $\partial M$, which is the only minimal surface in $(M, g)$. In this paper we investigate how large the mean curvature $H$ of embedded, constant mean curvature $(\mathrm{CMC})$ surface in the homology class of $\partial M$ can be. The main result in this paper is an upper bound for this curvature. Combined with an area estimate, we then show that there exists a CMC surface which attains this maximum.

The main motivation for this work are CMC foliations. These foliations have been used successfully in general relativity to study the center of mass of isolated systems [HY96] and the Riemannian Penrose inequality [Bra97. The existence result in [HY96] constructs a CMC foliation in the asymptotic region near infinity. The natural question arises how far to the interior these foliations can be extended. It is clear that in general topological reasons 
imply non-existence of an entire smooth foliation. This calls for a weak version of a CMC foliation.

Let us consider a different perspective. If the interior boundary $\partial M$ is an outermost minimal surface, that is $(M, g)$ does not contain any other minimal surface, then it is straightforward to construct a local CMC foliation near $\partial M$, cf. lemma 4.1. So another question is how far this interior foliation can be extended outward, away from $\partial M$. This is by far an easier question than extending the foliation inward.

The reason is the following. Roughly speaking, if we consider a potential CMC foliation reaching from $\partial M$ to infinity, then the mean curvature has to increase near $\partial M$, and decrease as in Euclidean space when approaching infinity, as the surfaces of the foliation enclose increasing volume with $\partial M$. This has two implications. First, there is a maximal value of CMC along this foliation, and second that there are two types of behavior. The first type is portions along which CMC increases and the other is where CMC decreases. The former includes the region near $\partial M$ and the latter the asymptotic region.

The region in which $\mathrm{CMC}$ increases is easier to handle, as the maximum principle implies that the CMC surfaces along the foliation can not touch. In the exterior region it is a lot harder to get control on the separation of the surfaces, as one can see from the many different foliations by spheres that are possible in $\mathbf{R}^{3}$.

This result gives a partial answer to the above questions. In section 3 we show that there is a bound for the maximal CMC of a surface homologous to $\partial M$. This needs a lower bound on the scalar curvature of $M,{ }^{M} \mathrm{Sc} \geq-C$, and uses the fact that $\partial M$ is area minimizing. The condition that the surface be homologous to $\partial M$ is necessary, as near maxima of the sectional curvature a CMC foliation exits where homologically trivial spheres have unbounded CMC [Ye91].

In section 4 the curvature bound is used to construct a surface which realizes the maximal CMC $H_{\max }$. This existence result needs the stronger assumption that ${ }^{M} \mathrm{Sc} \geq 0$, in order to ensure that the area of CMC surfaces is bounded. A unique such surface can be selected by demanding that it be the innermost such surface.

We show that this surface bounds a region with $\partial M$, which can be regarded as a manifold with boundary, cf. the discussion at the end of section 4 and in section 5. Using the outer boundary as barrier, we can construct an increasing family of sets bounded by surfaces with CMC ranging form 0 at the horizon to $H_{\max }$ at the boundary. This increasing family is a candidate 
for a weak version of a CMC foliation reaching up to the $H_{\max }$-surface. We explore some basic properties in the second half of section 5 .

\section{Preliminaries}

We consider asymptotically flat manifolds $(M, g)$ with an inner boundary $\partial M$ which is an outermost minimal surface. Such manifolds $M$ are called exterior regions. The requirement of asymptotic flatness means that there exists a compact set $K \subset M$ and a diffeomorphism $x: M \backslash K \rightarrow \mathbf{R}^{3} \backslash B_{1}(0)$ such that in the $x$-coordinates the metric $g$ approaches the Euclidean metric $\delta$, that is there exists $C$ such that

$$
r|g-\delta|+r^{2}|\partial g| \leq C
$$

To say that $\partial M$ is an outermost minimal surface means that there does not exist another minimal surface in $M$ which is homologous to $\partial M$. For an asymptotically flat manifold which contains minimal surfaces, the outermost minimal surface always exists and is unique [HI01, Section 4]. An exterior region $M$ is diffeomorphic to $\mathbf{R}^{3} \backslash\left(\bigcup_{i=1}^{N} B_{i}\right)$, where the $B_{i}$ are open balls with disjoint closure. Hence $\partial M=\bigcup_{i=1}^{N} S_{i}$, where $S_{i}=\partial B_{i}$. This restricted topology does not require any curvature assumptions. The fact that $\partial M$ is an outermost minimal surface implies furthermore that for each $I \subset\{1, \ldots, N\}$ the set $\bigcup_{i \in I} S_{i}$ minimizes area in its homology class, in particular $\partial M$ is minimizing.

Let $\Sigma \subset M$ be a two-sided surface. We assume that we can identify one side of $\Sigma$ as the outside, and denote the outward pointing normal by $\nu$. We denote by $\gamma$ the induced metric. The mean curvature $H=\operatorname{div} \nu$ is taken with respect to the outward pointing normal as is the second fundamental form $A$. By ${ }^{\Sigma}$ Sc we denote the scalar curvature of $\Sigma$. The trace free part of the second fundamental form will be denoted by $\AA=A-\frac{1}{2} H \gamma$.

Consider a normal variation of $\Sigma$, that is a map $F: \Sigma \times(-\varepsilon, \varepsilon) \rightarrow M$ with $F(\cdot, 0)=\operatorname{id}_{\Sigma}$ and $\left.\frac{d F}{d t}\right|_{=} f \nu$. The linearization of the operator which assigns the mean curvature to the surfaces $\Sigma_{t}=F(\Sigma, t)$ is given by

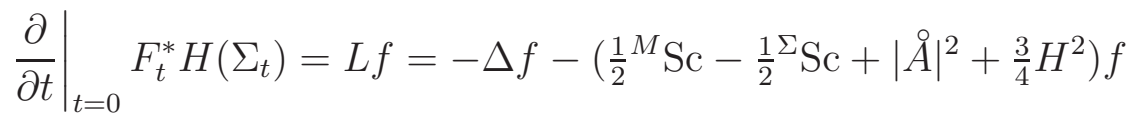

where $F_{t}=F(\cdot, t): \Sigma \rightarrow \Sigma_{t}$ and $\Delta$ denotes the Laplace-Beltrami operator along $\Sigma$. Here ${ }^{M}$ Sc and ${ }^{\Sigma}$ Sc denote the scalar curvature of $M$ and $\Sigma$. $L$ is called the stability operator, or Jacobi operator. 
When dealing with constant mean curvature surfaces $H=$ const, there are two types of stability discussed in the literature. The first notion is strong stability, where we assume that $L$ is a non-negative operator, that is

$$
\int_{\Sigma} f^{2}\left(\frac{1}{2}^{M} \mathrm{Sc}-\frac{1}{2}^{\Sigma} \mathrm{Sc}+\frac{1}{2}|\AA|^{2}+\frac{3}{4} H^{2}\right) \mathrm{d} \mu \leq \int_{\Sigma}|\nabla f|^{2} \mathrm{~d} \mu \quad \forall f \in C^{\infty}(\Sigma) .
$$

Here $\nabla f$ denotes the tangential gradient of $f$. Note that strong stability means that the principal eigenvalue of $L$, that is the smallest eigenvalue, is non-negative. The second notion, simply called stability comes from the fact that the constant mean curvature equation is the Euler-Lagrange equation for the isoperimetric problem, that is for minimizing the area of $\Sigma$, while keeping enclosed volume constant. Minimizers of this variational principle satisfy the stability inequality

$$
\begin{aligned}
\int_{\Sigma} f^{2}\left(\frac{1}{2}^{M} \mathrm{Sc}-\frac{1}{2}^{\Sigma} \mathrm{Sc}+\frac{1}{2}|\stackrel{\AA}{A}|^{2}+\frac{3}{4} H^{2}\right) \mathrm{d} \mu & \leq \int_{\Sigma}|\nabla f|^{2} \mathrm{~d} \mu \\
\forall f & \in C^{\infty}(\Sigma) \text { with } \int_{\Sigma} f \mathrm{~d} \mu=0 .
\end{aligned}
$$

Hence, strong stability implies stability, but not vice-versa. For the following discussion only strong stability plays a role.

Subsequently, we deal with surfaces which are not necessarily connected. We say that such a surface is strongly stable if each of its components is strongly stable, and thus if a surface is not strongly stable, it means that at least one of its components is not strongly stable.

The surfaces $\Sigma$ in question will be homologous to $\partial M$. In the case that $\Sigma$ does not touch $\partial M$ this means that there exists an open set $\Omega$ such that $\partial \Omega$ is the disjoint union $\partial \Omega=\partial M \cup \Sigma$. As we orient $\partial M$ with the normal pointing into $M$, the correct orientation of $\Sigma$ corresponds to the normal vector pointing out of $\Omega$. We will make this assumption subsequently without further notice.

\section{An upper bound for CMC}

This section is devoted to derive an upper bound for the constant mean curvature of a compact, smooth, embedded CMC surface homologous to $\partial M$. Note that $\Sigma$ need not be connected for the subsequent arguments. This upper bound only requires a lower bound on the scalar curvature of $M$, that is ${ }^{M} \mathrm{Sc} \geq-C$ for some $C \geq 0$. 
Before we can approach the main theorem, we review an existence theorem for prescribed mean curvature surfaces [AM07, Theorem 6.1]. This theorem implies the following existence theorem for strongly stable CMC surfaces.

Theorem 3.1. Let $(\Omega, g)$ be a compact Riemannian manifold with smooth boundary $\partial \Omega$ which is the disjoint union $\partial \Omega=\partial^{-} \Omega \cup \partial^{+} \Omega$, where $\partial^{ \pm} \Omega$ are smooth, non-empty and without boundary. Assume that $\partial^{-} \Omega$ has mean curvature $H^{-}$, where $H^{-}$is taken with respect to the normal pointing into $\Omega$, and $\partial^{+} \Omega$ has mean curvature $H^{+}$, where $H^{+}$is taken with respect to the normal pointing out of $\Omega$. Let $h$ be such that $\max _{\partial^{-} \Omega} H^{-} \leq h \leq \min _{\partial^{+} \Omega} H^{+}$. Then there exists a compact, smooth, embedded, strongly stable CMC surface $\Sigma \subset \Omega$, homologous to $\partial^{-} \Omega$ with $H(\Sigma)=h$.

Proof. The theorem is a direct consequence of Theorem 6.1 from AM07. This theorem states that if $(\Omega, g)$ is as in the assumption and $K$ is a symmetric bilinear form on $\Omega$, then if $\theta^{+}\left(\partial^{-} \Omega\right) \leq 0$ and $\theta^{+}\left(\partial^{+} \Omega\right) \geq 0$ then there exists a compact, smooth, embedded, surface $\Sigma$ homologous to $\partial^{-} \Omega$ with $\theta^{+}(\Sigma)=0$, which is stable in the sense of surfaces with $\theta^{+}=0$. Here $\theta^{+}=H+P$, where $H$ is the mean curvature as usual, and $P=\operatorname{tr}_{\Sigma} K=$ $\operatorname{tr}_{M} K-K(\nu, \nu)$ is the trace of $K$ restricted to $T \Sigma$.

We apply this theorem to the data $\left(\Omega, g, K=-\frac{1}{2} h g\right)$ such that for any surface $\Sigma$ we have $\theta^{+}(\Sigma)=H-h$. Thus $\theta^{+}\left(\partial^{-} \Omega\right)=H^{-}-h \leq 0$ and $\theta^{+}\left(\partial^{+} \Omega\right)=H^{+}-h \geq 0$, and the existence of a surface $\Sigma$ with $H(\Sigma)=h$ follows from the existence part of theorem 6.1 in [AM07]. The resulting surface is also stable in the sense of $\theta^{+}=0$ surfaces, which implies that the smallest eigenvalue of the operator

$$
\tilde{L} f=-\Delta f+2 S(\nabla f)-f\left(\operatorname{div} S-\frac{1}{2}\left|A+K^{\Sigma}\right|^{2}-|S|^{2}+\frac{1}{2}^{\Sigma} \mathrm{Sc}-\mu+J(\nu)\right)
$$

is non-negative. Here $K^{\Sigma}=\left.K\right|_{\Sigma}, S=K(\nu, \cdot)^{T}$, where $T$ denotes tangential projection, $\mu=\frac{1}{2}\left({ }^{M} \mathrm{Sc}-|K|^{2}+\left(\operatorname{tr}_{M} K\right)^{2}\right)$ and $J=\operatorname{div} K-{ }^{M} \nabla \operatorname{tr}_{M} K$. On a surface with $\theta^{+}=0$ we have for our choice of $K$ that $S=0, J=0$, $\left|A+K^{\Sigma}\right|^{2}=|A|^{2}-\frac{1}{2} H^{2}=|\AA|^{2}$ and $\mu=\frac{1}{2}^{M} \mathrm{Sc}+\frac{3}{4} H^{2}$. Thus we find that $\tilde{L}$ is nothing but the stability operator $L$ and non-negativity of its first eigenvalue means strong stability.

Remark 3.2. A similar existence theorem could be derived by analyzing the functional

$$
J_{h}(F)=\left|\partial^{*} F\right|-h \operatorname{Vol}(F)
$$

for sets $F$ with finite perimeter. 
Using the previous existence theorem together with the fact that $\partial M$ can be used as inner barrier, we infer the following lemma.

Lemma 3.3. If $\Sigma \subset M$ is a CMC surface with $H>0$ homologous to $\partial M$, then there exists a strongly stable CMC surface $\Sigma^{\prime}$ in the same homology class with $H\left(\Sigma^{\prime}\right)=H(\Sigma)$.

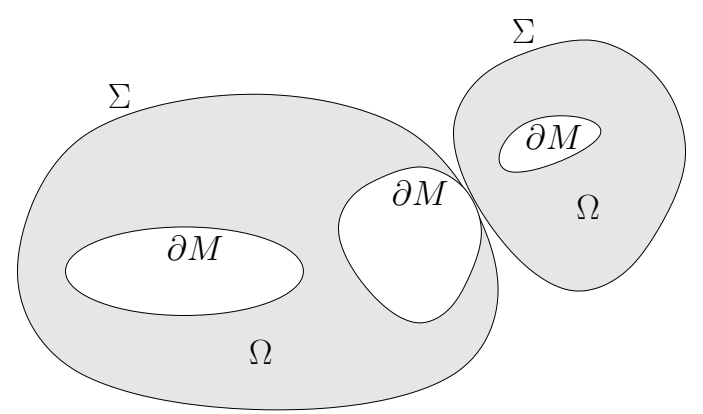

Figure 1: If a surface $\Sigma$ homologous to $\partial M$ intersects $\partial M$, then there is one component of $\Sigma$ which intersects $\partial M$ such that the outer normals point in the same direction.

Proof. First, note that $\Sigma$ can not touch $\partial M$, cf. figure1, As $\Sigma$ is homologous to $\partial M$, there exists a set $\Omega$ and a set $I \subset\{1, \ldots, N\}$ such that $\partial \Omega=$ $\bigcup_{i \in I} S_{i} \cup \Sigma_{0}$, and $\Sigma=\Sigma_{0} \cup \bigcup_{i \notin I} S_{I}$. As $H(\Sigma)>0$, we have must have $I=\{1, \ldots, N\}$, and thus $\Sigma_{0}=\Sigma$ and $\partial M \subset \Omega$. Thus if $\partial M$ and $\Sigma$ intersect, there exists a component $\Sigma_{1}$ of $\Sigma$ which intersects $\partial M$ at a point where the normals of $\Sigma_{1}$ and $\partial M$ point in the same direction. This is impossible, since the maximum principle would imply that $\Sigma_{1} \subset \partial M$.

Hence, $\Sigma$ lies completely in the interior of $M$ and we can apply theorem 3.1 with the so constructed $\Omega$ where $\partial M=\partial^{-} \Omega, \Sigma=\partial^{+} \Omega$ and $h=H(\Sigma)$. Thus we obtain $\Sigma^{\prime}$, a smooth, embedded strongly stable CMC surface.

Remark 3.4. Note that we can in fact show that the constructed $\Sigma^{\prime}$ can not touch any component of $\Sigma$ which is not strongly stable, as these components can be deformed in direction of $-\nu$, that is into $\Omega$ in such a way that their mean curvature increases.

It is now a simple matter to derive the claimed bound on the constant mean curvature from strong stability. As $\Sigma$ is not necessarily connected, we must make use of the fact that $\partial M$ is outermost to get a lower bound on the area of at least one component of $\Sigma$. 
Lemma 3.5. Let $(M, g)$ be asymptotically flat with inner boundary $\partial M$, which is an outermost minimal surface in $M$. Assume that

$$
{ }^{M} \mathrm{Sc} \geq-C
$$

Denote the components of $\partial M$ by $S_{i}, i=1, \ldots, N$ and let $A:=\max \left\{\left|S_{i}\right|\right.$ : $i=1, \ldots, N\}$. If $\Sigma \subset M$ is a CMC surface homologous to $\partial M$, then

$$
H(\Sigma)^{2} \leq \frac{16 \pi}{3 A}+\frac{2}{3} C
$$

Remark 3.6. An obvious modification yields a similar bound if $\Sigma$ is homologous to $\bigcup_{i \in I} S_{i}$, where $I \subset\{1, \ldots, N\}$ and $A$ is replaced by $A(I)=$ $\max _{i \in I}\left|S_{i}\right|$.

Proof. Assume that $A=\left|S_{1}\right|$. As $M$ is topologically equivalent to $\mathbf{R}^{3} \backslash$ $\bigcup_{i=1}^{N} B_{i}$, as explained in section 2, $\Sigma$ can be regarded as a surface embedded in $\mathbf{R}^{3}$. Then any component of $\Sigma$ bounds in $\mathbf{R}^{3}$, and since $\Sigma$ is homologous to $\bigcup_{i=1}^{N} S_{i}$, we infer that there exists one component $\Sigma_{1}$ of $\Sigma$ which is homologous to $S_{1} \cup \bigcup_{i \in J} S_{i}$, where $J \subset\{2, \ldots, N\}$ may be empty. Since $S_{1} \cup \bigcup_{i \in J} S_{i}$ is minimizing in its homology class in $M$ we find that $\left|\Sigma_{1}\right| \geq\left|S_{1} \cup \bigcup_{i \in J} S_{i}\right| \geq\left|\Sigma_{1}\right|=A$. Pick a test function $f \in C^{\infty}(\Sigma)$ with $f=1$ on $\Sigma_{1}$ and $f=0$ on all other components. Plugging $f$ into the strong stability inequality (2.1), we find that

$$
\int_{\Sigma_{1}} \frac{1}{2}|\stackrel{\AA}{A}|^{2}+\frac{3}{4} H^{2} \mathrm{~d} \mu \leq \int_{\Sigma_{1}} \frac{1}{2}^{\Sigma} \mathrm{Sc}-\frac{1}{2}^{M} \mathrm{Sc} \mathrm{d} \mu .
$$

From Gauss-Bonnet we infer that

$$
\int_{\Sigma_{1}} \frac{1}{2}^{\Sigma} \operatorname{Scd} \mu=4 \pi\left(1-\operatorname{genus}\left(\Sigma_{1}\right)\right) \leq 4 \pi .
$$

As $H$ is constant, combining the above inequality with the lower bound on ${ }^{M}$ Sc yields

$$
H^{2}\left|\Sigma_{1}\right| \leq \frac{16 \pi}{3}+\frac{2}{3} C\left|\Sigma_{1}\right|
$$

or after dividing by $\left|\Sigma_{1}\right|$,

$$
H^{2} \leq \frac{16 \pi}{3\left|\Sigma_{1}\right|}+\frac{2}{3} C
$$

which implies the claim, since $\left|\Sigma_{1}\right| \geq A$. 
Remark 3.7. The spatial Schwarzschild manifold of mass $m$ is $\left(\mathbf{R}^{3} \backslash\{0\}, \phi^{4} g^{e}\right)$ where $\phi=1+\frac{m}{2 r}$ and $g^{e}$ denotes the Euclidean metric on $\mathbf{R}^{3}$. It is scalar flat and if $m>0$ it has an outermost minimal surface at $r=\frac{m}{2}$. Thus $\left(\mathbf{R}^{3} \backslash B_{\frac{m}{2}}, \phi^{4} g^{e}\right)$ satisfies the assumptions of lemma 3.5 with $C=0$. The spheres $S_{r}(0)$ have constant mean curvature $H_{r}=\frac{2}{R} \frac{2 r-m}{2 r+m}$ where $R=\phi^{2} r$ is the geometric area radius of $S_{r}$ with respect to $g^{S}$, that is $\left|S_{r}\right|=4 \pi R^{2}$. $H_{r}$ assumes its maximum where $R=3 m$ and equals $\frac{2}{3 \sqrt{3} m}$ there. Thus, the estimate of equation (3.1) is sharp in this case, whereas the assertion of lemma 3.5 is not.

\section{Existence of surfaces with maximal CMC}

In this section we construct a surface with maximal constant mean curvature. In fact, for $(M, g)$ as before, we can let

$$
H_{\text {max }}:=\sup \{H(\Sigma): \Sigma \text { an embedded CMC surface homologous to } \partial M\} .
$$

As we have seen in the previous section, $H_{\max }$ is finite. Subsequently we show that $H_{\max }$ is attained at a strongly stable surface. We start by showing that $H_{\max }>0$.

Lemma 4.1. There exists a foliation of a neighborhood of $\partial M$ by $C M C$ surfaces $\Gamma_{s}, s \in[0, \varepsilon)$ with $H\left(\Gamma_{s}\right)>0$.

Proof. We construct the foliation near each component of $\partial M$ separately. Let $S_{i}$ be such a component. Note that $S_{i}$ is stable as a minimal surface, as $\partial M$ is outermost. If the principal eigenvalue $\lambda$ of $L$ on $S_{i}$ is positive, $\lambda>0$, then $L$ is invertible and we can construct a foliation of CMC surfaces by a simple application of the implicit function theorem.

Hence we can assume $\lambda=0$ from now on. Let $\phi>0$ denote the corresponding eigenfunction. In this case a CMC foliation can be constructed as in Gal06. We repeat the argument here for convenience. Consider the operator

$$
\mathcal{H}: C^{\infty}(\Sigma) \times \mathbf{R} \rightarrow C^{\infty} \times \mathbf{R}:(u, h) \mapsto\left(H(\operatorname{graph} u)-h, \int_{\Sigma} u \phi \mathrm{d} \mu\right)
$$

where graph $u=F_{u}(\Sigma)$ and $F_{u}(p)=\exp _{p}\left(u(p) \nu_{p}\right)$, where $p \in \Sigma$ and exp is the exponential map of $M$. Then $H$ (graph $u$ ) denotes the mean curvature of graph $u$ pulled-back to $\Sigma$ via $F_{u}$. 
We can compute the linearization of $\mathcal{H}$ at $(u, h)=(0,0)$ in direction $(v, s) \in$ $C^{\infty}(\Sigma) \times \mathbf{R}$ to be

$$
\mathcal{M}:=\left.D \mathcal{H}\right|_{(0,0)}(v, s)=\left(L v-s, \int_{\Sigma} v \phi \mathrm{d} \mu\right)
$$

Obviously $\mathcal{M}$ is invertible since $\operatorname{ker} L=\operatorname{span}\{\phi\}$ and the equation $L v=g$ is uniquely solvable if $\int_{\Sigma} g \phi \mathrm{d} \mu=0$ and $\int_{\Sigma} v \phi \mathrm{d} \mu=0$.

By the inverse function theorem, there exists $u(t)$ and $h(t)$ for small $t$ such that

$$
\mathcal{H}(u(t), h(t))=(0, t)
$$

This implies that the surfaces graph $u(t)$ have CMC. Differentiating equation (4.1) with respect to $t$ yields that

$$
\left(L u^{\prime}(0)-h^{\prime}(0), \int_{\Sigma} u^{\prime}(0) \phi \mathrm{d} \mu\right)=(0,1)
$$

and hence that $h^{\prime}(0) \in \operatorname{im} L \perp$ ker $L$, that is $\int h^{\prime}(0) \phi \mathrm{d} \mu=0$. Since $h^{\prime}(0)$ is a constant and $\phi>0$, we infer $h^{\prime}(0)=0$. Then $u^{\prime}(0) \in \operatorname{ker} L$ and $u^{\prime}(0)=\alpha \phi$ where $\alpha>0$, by (4.2). Thus, the graph $u(t)$ form a foliation near $S_{i}$.

As $\partial M$ is outermost, we must have that $h(t)>0$ for all $t$, and we thus found the foliation near $S_{i}$. As $h(t)$ is smooth, there exists a $t_{0}$ such that $h$ is increasing on $\left[0, t_{0}\right)$.

Thus we can find the required CMC foliation near each component of $\partial M$ separately and join it to give a CMC foliation near $\partial M$.

Remark 4.2. A different way to see that $H_{\max }>0$ is to use asymptotic flatness to conclude that there exists a surface in the asymptotic end with positive mean curvature. An application of theorem 3.1 then yields a CMC surface with positive CMC. However, the previous lemma emphasizes that not only the asymptotic behavior near infinity, but also the local geometry near $\partial M$ gives a lower bound on $H_{\max }$.

Standard arguments show that there are uniform bounds on the second fundamental form of strongly stable CMC surfaces.

Lemma 4.3. If $\Sigma$ is a strongly stable CMC surface then there exists a constant $C=C\left(\left\|^{M} \operatorname{Rm}\right\|_{C^{0}}, \operatorname{inj}(M, g)^{-1}, \sup _{\Sigma}|H|\right)$ such that

$$
\sup _{\Sigma}|A| \leq C
$$


Proof. First, there exists $0<r_{0}=r_{0}\left(\left\|{ }^{M} \mathrm{Rm}\right\|_{C^{0}}, \sup _{\Sigma}|H|\right)$ such that for all $r<r_{0}$ and $p \in \Sigma$ the area of the intrinsic balls $B^{\Sigma}(p, r)$ around $p$ with radius $r$ is bounded

$$
\left|B^{\Sigma}(p, r)\right| \leq 6 \pi r^{2}
$$

See for example [AM05, Theorem 8.1], which goes back to Pog81. With this local bound on area, the usual argument for deriving curvature bounds yields the desired estimate, cf. [SSY75, we refer to [AM05, Section 6] for a detailed proof in a slightly more general setting.

Before we can attempt the construction of surfaces realizing $H_{\max }$, we need a diameter bound for strongly stable CMC surfaces [Ros06].

Lemma 4.4. Let $(M, g)$ be a complete Riemannian 3-manifold with ${ }^{M} \mathrm{Sc} \geq 0$ and let $\Sigma \subset M$ be a closed, connected, strongly stable CMC surface with $H(\Sigma) \neq 0$. Then

$$
\operatorname{diam}(\Sigma) \leq \frac{2 \pi}{3 H}
$$

Proof. This estimate is a direct consequence of [Ros06, Theorem 1].

Theorem 4.5. Let $(M, g)$ be an asymptotically flat Riemannian manifold with ${ }^{M} \mathrm{Sc} \geq 0$ and a non-empty inner boundary $\partial M$, which is an outermost minimal surface. Assume that $\left\|^{M} \mathrm{Rm}\right\|_{C^{0}}$ is finite and $\operatorname{inj}(M, g)$ is non-zero. Then $H_{\max }$ is attained at a compact, immersed, strongly stable surface $\Sigma$ homologous to $\partial M . \Sigma$ is a union of spheres.

Proof. Let $\left\{\Sigma^{n}\right\}_{\{n \geq 1\}}$ be family of CMC surfaces homologous to $\partial M$ with

$$
H\left(\Sigma^{n}\right) \rightarrow H_{\max }
$$

We show that after suitable modification, the sequence $\Sigma^{n}$ allows the extraction of a convergent subsequence.

In view of lemma 3.3 we can assume that the $\Sigma^{n}$ are strongly stable. Due to lemma 4.1 we can furthermore assume that $H\left(\Sigma^{n}\right) \geq \varepsilon$ for some suitably chosen $\varepsilon>0$.

Fix an arbitrary $n$ and denote $\Sigma:=\Sigma^{n}$. Let $\Sigma_{j}$ be the components of $\Sigma$, $j=1, \ldots N_{\Sigma}$. For $j=1, \ldots, N_{\Sigma}$ let $f_{j}$ be the test function which is equal to 1 on the $\Sigma_{j}$ and 0 on the other components, and plug $f_{j}$ in the strong stability inequality (2.1). This yields that $\Sigma_{j}$ is a sphere, as $\int_{\Sigma_{j}} H(\Sigma)^{2} \mathrm{~d} \mu>0$. 
Let

$$
J:=\left\{j: \Sigma_{j} \text { does not bound a compact region on its inside }\right\}
$$

and delete all components $\Sigma_{j}$ from $\Sigma$ where $j \notin J$. The surface

$$
\Sigma^{\prime}:=\bigcup_{j \in J} \Sigma_{j}
$$

is homologous to $\partial M$ and thus separates $\partial M$ from infinity. Recall that $M$ is diffeomorphic to $\mathbf{R}^{3} \backslash \bigcup_{i=1}^{N} B_{i}$ and consider $\Sigma^{\prime} \subset \mathbf{R}^{3}$.

Let $U \subset \mathbf{R}^{3}$ be such that $\mathbf{R}^{3} \backslash U$ is the non-compact component of $\mathbf{R}^{3} \backslash \Sigma^{\prime}$. Note that $\partial U$ consists of a subset of the components of $\Sigma^{\prime}$. Indeed $\partial U=\Sigma^{\prime}$. Otherwise there exists one component $\Sigma_{j}$ not in $U$ which bounds a compact region $\Omega_{j}$ on its outside, relative to a subset of $\partial M$. This is clearly impossible as the boudary of $M^{\prime}:=M \backslash \Omega_{j}$ has $H\left(\partial M^{\prime}\right) \leq 0$ and $H\left(\partial M^{\prime}\right) \neq 0$. This would imply the existence of a minimal surface outside of $\partial M$, contradicting the assumption that $\partial M$ is an outermost minimal surface.

Then $\Sigma^{\prime}:=\partial U$ has at most $N$ components, each of which is homologous to $\bigcup_{I_{j}} S_{i}$, where $I_{j} \subset\{1, \ldots, N\}$ is non-empty, and $I_{j} \cap I_{j^{\prime}}=\emptyset$ for $j \neq j^{\prime}$. To see this, let $U_{j}$ be the compact region in $\mathbf{R}^{3}$ bounded by $\Sigma_{j}$. Then $U_{j}$ contains at least one of the $B_{i}$, so it is clear that $I_{j} \neq \emptyset$. Since we have $\Sigma^{\prime}=\partial U$, no component of $\Sigma^{\prime}$ is separated from infinity by another component of $\Sigma^{\prime}$, in particular the outer normal direction of $\Sigma^{\prime}$ agrees with the outer normal to $\partial U$. As all the $B_{i}$ are contained in $U$ and the components of $\Sigma^{\prime}$ can not intersect, this implies that the each $B_{i}$ can be in at most one $U_{j}$. Thus the $I_{j}$ are mutually disjoint. For subsequent use we relabel the $\left(\Sigma^{n}\right)^{\prime}$ as $\Sigma^{n}$.

We thus have constructed a sequence $\left\{\Sigma^{n}\right\}$ of CMC surfaces with $H\left(\Sigma^{n}\right) \rightarrow$ $H_{\text {max }}$, where each of the $\Sigma^{n}$ is a strongly stable CMC surface with at most $N$ components, and each component is homologous to a non-empty union of components of $\partial M$.

As $H\left(\Sigma^{n}\right) \geq \varepsilon$, lemma 4.4 implies that each component of $\Sigma^{n}$ has bounded diameter. Such a component of $\Sigma^{n}$ encloses at least one of the $S_{i}$. We thus infer that there exists a compact set $B \subset M$ such that $\Sigma^{n} \subset B$ for all $n$. Furthermore, the curvature estimates from lemma 4.3 imply uniform curvature bounds for $\Sigma^{n}$. Therefore the Ricci curvature of $\Sigma_{n}$ is bounded below and standard volume comparison shows that each component of $\Sigma^{n}$ has bounded area. As there are at most $N$ components the $\Sigma^{n}$ have uniformly bounded area.

These three estimates, area, curvature, and the fact that the $\Sigma_{n}$ are contained in a compact set, imply that there exists a convergent subsequence 


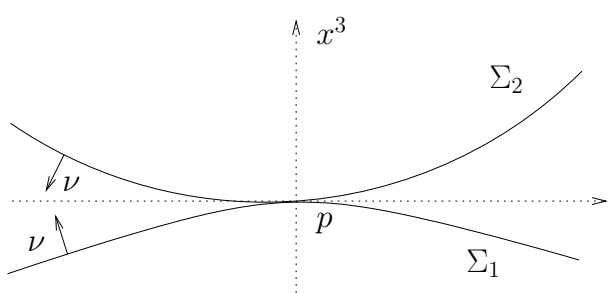

Figure 2: Two sheets touching on the outside.

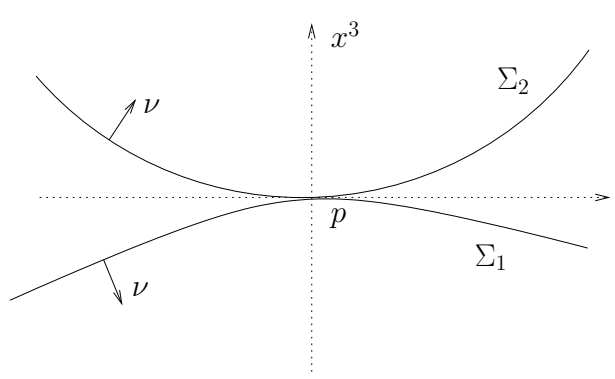

Figure 3: Two sheets touching on the inside.

and a limiting surface $\Sigma$, which has $\mathrm{CMC}$ and consists of strongly stable components. Note that the limit $\Sigma$ might not be embedded. Nevertheless, $\Sigma$ has an outward pointing normal vector field $\nu$ which is the limit of the outward pointing normal vector fields of the subsequence of $\Sigma_{n}$.

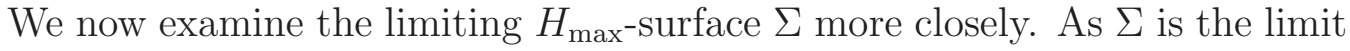
of embedded surfaces, $\Sigma$ can fail to be embedded only if $\Sigma$ touches itself. Let $p \in M$ denote such a point. Then at $p$ multiple sheets of $\Sigma$ can come together. Since we have bounded curvature and bounded area, there are at most finitely many such sheets $\Sigma_{k}^{p}, k=1, \ldots n(p)$, as each sheet takes up some area.

Around $p$ there are coordinates $\left\{x^{i}\right\}$ of $M$ such that the $\Sigma_{k}^{p}$ are $C^{\infty}$ graphs over an open subset $U^{p}$ of the $x^{1}, x^{2}$-plane. That is $\Sigma_{k}^{p}=\left\{x: x^{3}=u\left(x^{1}, x^{2}\right)\right\}$. We can assume that $u_{k} \leq u_{l}$ whenever $k \leq l$ since $\Sigma$ is the limit of embedded surfaces which bound a region with respect to $\partial M$. Each of the sheets comes with a normal vector field $\nu^{p}$ with respect to which $H=H_{\max }$. This can be either the downward or upward pointing normal to graph $u_{\tau}$, and this direction alternates.

We say that two sheets $\Sigma_{1}$ and $\Sigma_{2}$ touch on the outside at a point $p$, if the representing functions $u_{1} \leq u_{2}$ of these sheets are so that the outward normal of $\Sigma$ points upward along $u_{1}$ and downward along $u_{2}$, cf. figure 2 . On the other hand, if the normal along $u_{1}$ points downward, and upward along $u_{2}$, we say that $\Sigma$ touches itself on the inside.

The following theorem is a direct consequence of the strong maximum principle for surfaces with prescribed mean curvature.

Theorem 4.6. Let $\Sigma$ be the $H_{\max }$ surface constructed in theorem 4.5. Then if $\Sigma$ is not embedded, $\Sigma$ can only touch itself on the outside, and no more than two sheets of $\Sigma$ can meet at one point of $M$. 
Proof. Let $\Sigma_{1}$ and $\Sigma_{2}$ be two sheets of $\Sigma$ which meet on the inside, and let $u_{1} \leq u_{2}$ be the representing functions as described above. Instead of the upward normal, consider $u_{2}$ equipped with the downward normal. Then the mean curvature of graph $u_{2}$ is $-H_{\max }<0$ with respect to the downward normal, and the mean curvature of graph $u_{1}$ is $H_{\max }$ with respect to the downward unit normal. As graph $u_{1}$ and graph $u_{2}$ touch, we immediately obtain a contradiction to the strong maximum principle.

At any point in $M$ where three sheets of $\Sigma$ meet, $\Sigma$ must touch itself on the inside, thus this is ruled out by the above argument.

We now want to add a few remarks about the uniqueness of the $H_{\text {max }}$ surfaces. Indeed, we can single out one particular $H_{\text {max }}$-surface in $(M, g)$ by choosing the innermost $H_{\max }$ surface.

Theorem 4.7. Let $(M, g)$ be as in theorem 4.5. Then there exists a unique innermost surface in $(M, g)$ which is homologous to $\partial M$ and has $C M C H_{\max }$. The assertion of theorem 4.6 holds for $\Sigma$.

Proof. The construction of this surface is similar to the construction of the outermost MOTS in [AM07, Section 7]. Thus we mention only the key points for the construction.

Compactness. As in the proof of theorem 4.5, we infer compactness of the class of $H_{\text {max-surfaces by throwing away components which bound compact }}$ regions.

Monotonicity. Let $\Sigma_{i}$, with $i=1,2$ be two $H_{\max }$-surfaces for which the assertion of theorem 4.6 holds, and which are homologous to $\partial M$ and bound sets $\Omega_{i}$ with $\partial M$. Then $\Omega_{1} \cap \Omega_{2}$ contains a strongly stable $H_{\max }$-surface $\Sigma$ homologous to $\partial M$ which satisfies the assertion of theorem 4.6.

Monotonicity allows us to construct a sequence of surfaces $\Sigma_{k}$ bounding $\Omega_{k}$ together with $\partial M$, such that the $\Omega_{k}$ are decending. By compactness we find a limiting set $\Omega_{\infty}$ bounded by an $H_{\max }$-surface $\Sigma_{\infty}$ and $\partial M$.

\section{A proposal for a weak CMC foliation}

In this section we propose a weak version of a foliation by CMC surfaces of the interior region of $(M, g)$. There is more than one way to introduce such a foliation, and it is not clear whether the possibility discussed below is best suited for applications. 
Let $(M, g)$ be asymptotically flat with $\partial M$ an outermost minimal surface. Assume that ${ }^{M} \mathrm{Sc} \geq 0$ and let $\Sigma$ be the $H_{\max }$-surface homologous to $\partial M$ constructed in section 4 . We denote by the interior $\Omega$ of $M$ the components of

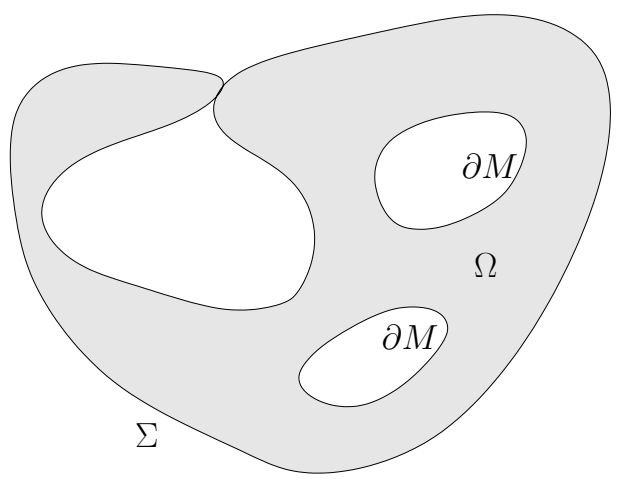

Figure 4: The interior region $\Omega$.

$M \backslash \Sigma$ which meet components of $\partial M$, cf. figure 4. As $\Sigma$ does not touch itself on the inside, $\Omega$ can be equipped with the structure of a smooth manifold with boundary $\partial M \cup \Sigma$, where we identify $\Sigma$ and $\partial M$ with the points added by the metric completion of $\Omega$. In this way, we separate the points of $\Sigma$ which are mapped to the same point in the immersion of $\Sigma$ into $M$. Note that the interior $\Omega$ is not a submanifold with smooth boundary in $M$ if $\Sigma$ is not embedded. The boundary of $\Omega$ consists of $\partial M$ on the inside, subsequently denoted by $\partial^{-} \Omega$, and $\Sigma$ on the outside, subsequently denoted by $\partial^{+} \Omega$.

\section{Construction}

To construct a weak CMC foliation for this new manifold $\Omega$, we introduce the following notion.

Definition 5.1. Let $\Sigma \subset \Omega$ be a smooth, embedded surface homologous to $\partial^{-} \Omega$, with $C M C h \in\left(0, H_{\max }\right)$. Denote by $U$ the region bounded by $\Sigma$ and $\partial^{+} \Omega$. If there does not exist a smooth embedded surface $\Sigma^{\prime}$ in $U$ with the same CMC h, then $\Sigma_{h}$ is called outermost.

By [AM07, Section 7], for each $h \in\left(0, H_{\max }\right)$ there exists a smooth, embedded surface $\Sigma_{h}$, homologous to $\partial^{-} \Omega$, which has CMC $h$ and is outermost in the sense of definition 5.1. We denote by $\Omega_{h}$ the open region bounded by $\partial^{-} \Omega$ and $\Sigma$. We define this family of sets to be the candidate for our weak $\mathrm{CMC}$ foliation of $\Omega$.

A useful side-effect of this definition is that the constructed sets are related to a variational principle. Consider sets $F$ of finite perimeter in $\Omega$. We will 
assume that $F \supset \Omega_{h}$ for some $h>0$. Thus $F$ has one boundary component which agrees with $\partial^{-} \Omega$. In accordance with the above notation, we denote by $\partial^{+} F$ the reduced boundary of $F$ without $\partial^{-} \Omega$, that is $\partial^{+} F=\partial^{*} F \cap \Omega$.

\section{Basic properties}

Consider the functional $J_{h}$, defined on the collection of sets $F$ of bounded perimeter in $\Omega$,

$$
J_{h}(F):=\left|\partial^{+} F\right|-h \operatorname{Vol}(F) .
$$

The critical points of $J_{h}$ are surfaces with $\mathrm{CMC} h$, so it is natural to consider this functional here.

We say that a set $E$ minimizes $J_{h}$ on the outside, if for all sets $F \supset E$ we have

$$
J_{h}(E) \leq J_{h}(F)
$$

Lemma 5.2. For each $h \in\left(0, H_{\max }\right)$, the set $\Omega_{h}$ defined above minimizes $J_{h}$ on the outside.

Proof. If $\Omega_{h}$ does not minimize $J_{h}$ on the outside, then there exits a minimizer $E_{h}$ for $J_{h}$ outside of $\Omega_{h}$, with $E_{h} \neq \Omega_{h}$. The outer boundary $E_{h}$ is a $C^{1, \alpha}$-surface, satisfying $H \geq h$ in a distributional sense, cf. HI01, Theorem 1.3]. It is smooth with $H=h$ where it does not touch $\Sigma_{h}$. By the strong maximum principle (which applies here as $\partial^{+} E_{h}$ is $C^{1, \alpha}$, see also [ZZ98, Section 3]) all components of $\partial^{+} E_{h}$ touching $\Sigma_{h}$ are contained in $\Sigma_{h}$. Thus, $\partial^{+} E_{h}$ is a smooth surface with CMC $h$ and lies on the outside of $\Sigma_{h}$. As $\Sigma_{h}$ is outermost, $E_{h}=\Omega_{h}$ as claimed.

This lemma implies that $\Sigma_{h}$ minimizes area on the outside.

Lemma 5.3. For all $h \in\left(0, H_{\max }\right)$ and all sets of finite perimeter $\Omega_{h} \subset F \subset$ $\Omega$, we have

$$
\left|\Sigma_{h}\right| \leq\left|\partial^{+} F\right|,
$$

in particular

$$
\left|\Sigma_{h}\right| \leq\left|\partial^{+} \Omega\right| .
$$


Proof. As $\Omega_{h}$ minimizes $J_{h}$ on the outside,

$$
\left|\Sigma_{h}\right|+h(\operatorname{Vol}(F)-\operatorname{Vol}(\Omega)) \leq\left|\partial^{+} F\right| .
$$

To conclude, we mention two other properties, which follow from the construction.

Lemma 5.4. 1. The sets $\Omega_{h}$ are increasing, that is $\Omega_{h_{1}} \subset \Omega_{h_{2}}$ if $h_{1}<h_{2}$.

2. If $h \in\left(0, H_{\max }\right)$ is fixed and $h_{k} \in\left(0, H_{\max }\right)$ a sequence with $h_{k} \geq h$ and $\lim _{k} h_{k}=h$, then

$$
\bar{\Omega}_{h}=\bigcap_{k \geq 1} \Omega_{h_{k}} .
$$

Here $\bar{\Omega}_{h}$ denotes the closure of $\Omega_{h}$ in $\Omega$.

Proof. Property 1 follows from theorem 3.1, as we can always use $\Sigma_{h_{1}}$ and $\partial^{+} \Omega$ as inner and outer barriers for the construction of a surface with CMC $h_{2}$ outside. Note that this requires the strong maximum principle to conclude that $\Sigma_{h_{1}}$ is disjoint from $\partial^{+} \Omega$.

To prove property 2, note that clearly $\bar{\Omega}_{h} \subset \bigcap_{k \geq 1} \Omega_{h_{k}}$, as there is a positive distance between $\Sigma_{h}$ and $\Sigma_{h^{\prime}}$ if $h<h^{\prime}$. On the other hand, in view of the curvature bound on $\Sigma_{h}$ and the area estimate, lemma 5.3, we can assume that the $\Sigma_{h_{k}}$ converge to a smooth surface $\Sigma^{\prime}$ with CMC $h$. By construction, $\Sigma^{\prime}$ lies on the outside of $\Sigma_{h}$ and hence must agree with $\Sigma_{h}$, as $\Sigma_{h}$ is outermost.

\section{Level set formulation}

Clearly, the sets $\Omega_{h}$ constructed above can be recognized as the sub-level sets of a function $u$. For $x \in \Omega$, we can define $u(x)$ as follows

$$
u(x):=\inf \left\{h: x \in \Omega_{h}\right\} .
$$

We denote the sub-level sets by

$$
\begin{aligned}
E_{h} & :=\{x \in \Omega: u(x)<h\}, \text { and } \\
E_{h}^{+} & :=\{x \in \Omega: u(x) \leq h\} .
\end{aligned}
$$

We can say the following about these level sets. 
Lemma 5.5. For all $h \in\left[0, H_{\max }\right]$ we have that $E_{h} \subset \Omega_{h}$ and $E_{h}^{+}=\bar{\Omega}_{h}$.

Proof. If $x \in E_{h}$ then $u(x)<h$ which implies $x \in \Omega_{h}$ by the definition of $u$, hence $E_{h} \subset \Omega_{h}$.

Let $x \in E_{h}^{+}$, that is $u(x) \leq h$. Then for all $h^{\prime}>h$ we have that $x \in \Omega_{h^{\prime}}$. As the intersection of all $\Omega_{h^{\prime}}$ with $h^{\prime}>h$ is $\bar{\Omega}_{h}$ by property 2 of lemma 5.4, we infer that $E_{h}^{+} \subset \bar{\Omega}_{h}$. To see the other inclusion, note that if $x \in \bar{\Omega}_{h}$ then $x \in \Omega_{h^{\prime}}$ for all $h^{\prime}>h$.

Lemma 5.6. If $u$ is as defined above, then $u \in B V(\Omega) \cap C^{0}(\Omega)$, where $B V(\Omega)$ denotes the space of functions with bounded variation and $C^{0}(\Omega)$ denotes the space of bounded continuous functions.

Proof. First note that $u(x) \in\left[0, H_{\text {max }}\right]$ and thus $u$ is bounded.

We show that $u$ is continuous. First, note that since $E_{h}^{+}$is closed, we have that $\{u>h\}=\Omega \backslash E_{h}^{+}$is open. Furthermore

$$
\{u=h\}=\bar{\Omega}_{h} \backslash \bigcup_{h^{\prime}<h} \Omega_{h^{\prime}}
$$

hence $\{u<h\} \bigcup_{h^{\prime}<h} \Omega_{h^{\prime}}$ and thus $\{u<h\}$ is also open. These two properties imply the continuity of $u$.

Furthermore, for all $k \in \mathbf{N}$ we can choose values $0=h_{0}^{k}<\ldots<h_{N(k)}^{K}=$ $H_{\max }$ such that $\left|h_{i}^{k}-h_{i-1}^{k}\right|<1 / k$ for $i=1, \ldots, N(k)$. Let

$$
u_{k}:=\sum_{i=1}^{N(k)}\left(h_{i}^{k}-h_{i-1}^{k}\right) \chi_{E_{h_{i}^{k}}^{+}},
$$

where $\chi_{E}$ denotes the characteristic function of a set $E$. Note that the $u_{k}$ converge uniformly to $u$ as $k \rightarrow \infty$ since $u$ is continuous. Furthermore, all $u_{k}$ have their $B V$-norm bounded by $\left|\Sigma_{H_{\max }}\right| H_{\max }$, and thus contain a subsequence that converges weakly to a limit $u_{\infty} \in B V$. As the $u_{k}$ converge uniformly to $u$ we have that $u=u_{\infty}$ and hence $u$ is in $B V$ and has $B V$ norm bounded by $\left|\Sigma_{H_{\max }}\right| H_{\max }$.

In HI01, Huisken and Ilmanen introduced a notion of weak solutions to the level-set inverse mean curvature flow. This notion motivates the following definition of a self-referencing functional on sets $F$ of bounded variation.

$$
J_{u}(F):=\left|\partial^{+} F\right|-\int_{F} u d x .
$$

Based on this functional we introduce the notion of weak CMC foliations. 
Definition 5.7. We say that $u$ is a weak (respectively sub-, super-) solution to the CMC foliation problem, if the sets $E_{h}^{+}:=\{x \in M: u(x) \leq h\}$ minimize $J_{u}$ (from the outside, inside respectively).

With respect to the above definition, we show the following theorem.

Theorem 5.8. The function $u$, as defined above, is a weak sub-solution to the $C M C$ foliation problem.

Proof. Let $F \supset \bar{\Omega}_{h}$ be any subset of finite perimeter. Fix $\varepsilon>0$ and pick $h_{i} \in\left(0, H_{\max }\right)$ such that

$$
h=h_{0}<h_{1}<\ldots<h_{N}
$$

$h_{i}-h_{i-1}<\varepsilon$ and $h_{N}$ is such that $F \subset \Omega_{h_{N}}$. For each $h_{i}$ we know that $\Omega_{h_{i}}$ minimizes $J_{h_{i}}$ from the outside. Hence we can compare with the set $F_{i}:=\left(\Omega_{h_{i+1}} \cap F\right) \cup \bar{\Omega}_{h_{i}}$ and find that

$$
J_{h_{i}}\left(\Omega_{h_{i}}\right) \leq J_{h_{i}}\left(F_{i}\right)
$$

Expanding this out, we obtain

$$
\begin{aligned}
\left|\Sigma_{h_{i}}\right|-h_{i} \operatorname{Vol}\left(\Omega_{h_{i}}\right) \leq & \left|\partial^{+} F \cap\left(\bar{\Omega}_{h_{i+1}} \backslash \bar{\Omega}_{h_{i}}\right)\right|+\left|\Sigma_{h_{i+1}} \cap F\right|+\left|\Sigma_{h_{i}} \backslash F\right| \\
& -h_{i} \operatorname{Vol}\left(\Omega_{h_{i}}\right)-h_{i} \operatorname{Vol}\left(F \cap\left(\bar{\Omega}_{h_{i+1}} \backslash \bar{\Omega}_{h_{i}}\right)\right)
\end{aligned}
$$

sorting terms, this implies that

$$
\left|\Sigma_{h_{i}} \cap F\right|-\left|\Sigma_{h_{i+1}} \cap F\right| \leq\left|\partial^{+} F \cap\left(\bar{\Omega}_{h_{i+1}} \backslash \bar{\Omega}_{h_{i}}\right)\right|-h_{i} \operatorname{Vol}\left(F \cap\left(\bar{\Omega}_{h_{i+1}} \backslash \bar{\Omega}_{h_{i}}\right)\right) .
$$

Taking the sum, we find that

$$
\sum_{i=0}^{N-1}\left(\left|\Sigma_{h_{i}} \cap F\right|-\left|\Sigma_{h_{i+1}} \cap F\right|\right) \leq\left|\partial^{+} F\right|-\sum_{i=0}^{N-1} h_{i} \operatorname{Vol}\left(F \cap\left(\bar{\Omega}_{h_{i+1}} \backslash \bar{\Omega}_{h_{i}}\right)\right) .
$$

Since $\Omega_{h} \subset F$ and $F \subset \bar{\Omega}_{h_{N}}$ we have that $\left|\Sigma_{h_{0}} \cap F\right|=\left|\Sigma_{h}\right|$ and $\left|\Sigma_{h_{N}} \cap F\right|=0$. So the above implies

$$
\left|\Sigma_{h}\right| \leq\left|\partial^{+} F\right|-\sum_{i=0}^{N-1} h_{i} \operatorname{Vol}\left(F \cap\left(\bar{\Omega}_{h_{i+1}} \backslash \bar{\Omega}_{h_{i}}\right)\right)
$$


As $h_{i} \leq u \leq h_{i+1}$ on $\Omega_{h_{i+1}} \backslash \Omega_{h_{i}}$ we can estimate

$$
\begin{aligned}
\int_{F} u d x-\int_{\Omega_{h}} u d x & =\sum_{i=0}^{N-1} \int_{F \cap\left(\Omega_{h_{i+1}} \backslash \Omega_{h_{i}}\right)} u d x \\
& \leq \sum_{i=0}^{N-1} h_{i+1} \operatorname{Vol}\left(F \cap\left(\Omega_{h_{i+1}} \backslash \Omega_{h_{i}}\right)\right) \\
& \leq \sum_{i=0}^{N-1}\left(h_{i}+\varepsilon\right) \operatorname{Vol}\left(F \cap\left(\Omega_{h_{i+1}} \backslash \Omega_{h_{i}}\right)\right) \\
& \leq \sum_{i=0}^{N-1} h_{i} \operatorname{Vol}\left(F \cap\left(\Omega_{h_{i+1}} \backslash \Omega_{h_{i}}\right)\right)+\varepsilon \operatorname{Vol}\left(F \backslash \Omega_{h}\right) .
\end{aligned}
$$

Combining this estimate with equation (5.1) from above, we arrive at

$$
\int_{F} u d x-\int_{\Omega_{h}} u d x \leq\left|\partial^{+} F\right|-\left|\Sigma_{h}\right|+\varepsilon \operatorname{Vol}\left(F \backslash \Omega_{h}\right)
$$

This implies that

$$
J_{u}\left(\Omega_{h}\right) \leq J_{u}(F)+\varepsilon \operatorname{Vol}\left(F \backslash \Omega_{h}\right)
$$

as $\varepsilon$ was arbitrary, this yields the claim.

Remark 5.9. We arrived at a weak sub-solution to the CMC foliation problem in the interior region by taking the outermost sets with curvature $\Omega_{h}$. Analogously, we can construct the sets $\tilde{\Omega}_{h}$ bounded by the innermost surfaces with CMC $h$. Then the procedure above will result in surfaces minimizing $J_{h}$ from the inside, which in turn implies that the corresponding level set function $\tilde{u}$ is a super-solution to the weak CMC foliation problem.

Having these sub- and super- solutions at hand it should be possible to construct a weak solution of the CMC foliation problem in the sense as defined above. This is research in progress, details of which will appear elsewhere.

\section{Acknowledgements}

Research on this project started while the author was visiting Stanford University. During this time, the author also received financial support from the Alexander-von-Humboldt foundation. 


\section{References}

[AM05] L. Andersson and J. Metzger, Curvature estimates for stable marginally trapped surfaces, arXiv:gr-qc/0512106, 2005.

[AM07] - The area of horizons and the trapped region, arXiv:0708.4252 [gr-qc], 2007.

[Bra97] H. L. Bray, The Penrose inequality in General Relativity and volume comparison theorems involving scalar curvat ure, Ph.D. thesis, Stanford University, 1997.

[Gal06] Gregory J. Galloway, Rigidity of outer horizons and the topology of black holes, arXiv:gr-qc/0608118, 2006.

[HI01] G. Huisken and T. Ilmanen, The inverse mean curvature flow and the Riemannian Penrose inequality, J. Differential Geom. 59 (2001), no. 3, 353-437.

[HY96] G. Huisken and S.-T. Yau, Definition of center of mass for isolated physical systems and unique foliations by stable spheres with constant mean curvature, Invent. Math. 124 (1996), no. 1-3, 281-311.

[Pog81] A. V. Pogorelov, On the stability of minimal surfaces, Dokl. Akad. Nauk SSSR 260 (1981), no. 2, 293-295.

[Ros06] Harold Rosenberg, Constant mean curvature surfaces in homogeneously regular 3-manifolds, Bull. Austral. Math. Soc. 74 (2006), no. 2, 227-238. MR MR2260491 (2007g:53009)

[SSY75] R. Schoen, L. Simon, and S. T. Yau, Curvature estimates for minimal hypersurfaces, Acta Math. 134 (1975), no. 3-4, 275-288.

[Ye91] Rugang Ye, Foliation by constant mean curvature spheres, Pacific J. Math. 147 (1991), no. 2, 381-396. MR MR1084717 (92f:53030)

[ZZ98] W. P. Ziemer and K. Zumbrum, The obstacle problem for functions of least gradient, Mathematica Bohemica 124 (1998), 193-219. 